\title{
Efficient Simulation for Fixed-Receiver Bistatic SAR with Time and Frequency Synchronization Errors
}

\author{
Feifei YAN, Wenge CHANG, Xiangyang LI \\ College of Electronic Science and Engineering, National University of Defense Technology, \\ Changsha, Hunan, P. R. China, 410073 \\ ddx_yff@163.com, changwenge@nudt.edu.cn, 1xyniu@sina.com
}

\begin{abstract}
Time and frequency synchronization is the key technique of bistatic synthetic aperture radar(BiSAR) system, and raw data simulation is an effective tool for verifying the time and frequency synchronization techniques. According to the two-dimensional (2-D) frequency spectrum of fixedreceiver BiSAR with time and frequency synchronization errors, a rapid raw data simulation method is proposed in this paper. Through 2-D inverse Stolt transform in 2-D frequency domain and phase compensation in range-Doppler frequency domain, this method can realize 2-D spatial variation simulation for fixed-receiver BiSAR with time and frequency synchronization errors in a reasonable time consumption. Then the simulation efficiency of scene raw data can be significantly improved. Simulation results of point targets and extended scene are presented to validate the feasibility and efficiency of the proposed simulation method.
\end{abstract}

\section{Keywords}

Simulation, BiSAR, fixed-receiver, inver Stolt transform, time and frequency synchronization errors

\section{Introduction}

SAR is an imaging tool for remote sensing. It can work day-and-night in all weather conditions and acquire microwave images of the Earth's surface with high resolution, which makes it unsubstitutable for surface surveillance. BiSAR is characterized by placing transmitter and receiver on separate platforms. By using appropriate bistatic configurations, it can achieve additional benefits like improving feature extraction and classification of targets, increasing surveillance for applications in conflict areas, etc. However, such a spatial separation results in problems and special requirements that are either not encountered or encountered in less serious form for monostatic SAR. A problem common to most bistatic radar systems is time and frequency synchronizations [1].

Raw data simulation can be useful for studying the effects of time and frequency synchronization errors in BiSAR and testing the synchronization algorithms. In order to eas- ily test the effectiveness of such algorithms, simple point target simulations are usually employed. However, when image ambiguity and interferometric processing methods are concerned, scene raw data simulation have to be performed. Scene raw data can be simulated by means of time domain approach according to the geometry of the SAR system. However, as the raw data of simulated scene is calculated target by target (TBT) and pulse by pulse in 2-D time domain (range and azimuth dimensions) [2], the computational efficiency is very low, especially when large size of scene is needed. Therefore, the efficiency should be improved, e.g., using frequency domain method which is based on 2-D frequency spectrum of the fixed-receiver BiSAR. As to rapid raw data simulation for fixed-receiver BiSAR with time and frequency synchronization errors, the difficulties come from two aspects. One is the simulation of 2-D spatial variation derived from that this system falls into translational variant case [3]. The other is how to introduce time and frequency synchronization errors in the raw data.

Different approaches for rapid raw data simulation have been proposed in recent years. Zhang [4] realized rapid raw data simulation for monostatic SAR by a special GPU workstation. The computing speed is fast, but the cost is high and the expansibility is poor. Franceschetti et al. [5-8] have done a lot of work on rapid raw data simulation, and proposed a series of simulator for rapid raw data simulation, such as 2-D Fourier domain approach and one-dimensional range Fourier domain approach. But the simulators involve approximations only acceptable in monostatic SAR and translational invariant BiSAR, so it cannot be applied in fixed-receiver BiSAR. Qiu et al. [2,9] studied the 2-D frequency domain model of monostatic SAR and translational invariant BiSAR. Through inverse Stolt transform, they realized rapid raw data simulation in 2-D frequency domain. However, because of the premise that the simulated BiSAR system is an ideal translational invariant case, this method cannot realize 2-D spatial variation simulation for fixed-receiver BiSAR with time and frequency synchronization errors. In conclusion, few studies are reported on rapid raw data simulation for fixed-receiver BiSAR with time and frequency synchronization errors.

Based on inverse Stolt transform in 2-D frequency domain and phase compensation in range-Doppler frequency domain, a rapid raw data simulation method for fixed-receiver 
BiSAR with time and frequency synchronization errors is proposed in this paper. Without computing the scene raw data by the TBT method in time domain, the proposed method can realize 2-D spatial variation simulation for fixed-receiver BiSAR with time and frequency synchronization errors in 2-D frequency domain, and then the simulation efficiency can be significantly improved. Meanwhile, raw data with synchronization errors generated by the proposed method can be well used for verifying the time and frequency synchronization techniques. This paper is arranged as follows: Section 2 introduces the echo model of fixed-receiver BiSAR with time and frequency synchronization errors. Section 3 gives the rapid raw data simulation method for fixed-receiver BiSAR. Raw data of point target and scene are presented in Section 4 to verify the proposed method. Finally, some conclusions are given in Section 5.

\section{Echo Model}

Scene raw data can be generated by the TBT method in time domain according to the geometry of fixed-receiver BiSAR, but it is too time-consuming to implement. Rapid raw data simulation can be achieved by using frequency domain approach which is based on 2-D frequency spectrum of the system. In this section, we will first introduce the echo model of fixed-receiver BiSAR with time and frequency synchronization errors, and then 2-D frequency spectrum of the echo is presented.

\subsection{Time and Frequency Synchronization Errors}

As antenna, oscillator, frequency synthesizer and timer are not shared in bistatic radar, there are a number of synchronization challenges that have to be overcome in the design and implementation $[10,11]$. Synchronizations include space synchronization, time synchronization and frequency synchronization. In this section, the time and frequency synchronization problem is discussed.

1) Time synchronization errors

The transmitted signal is defined by:

$$
s_{T}\left(\tau, t_{m}\right)=W_{r}(\tau) \exp (\varphi(\tau))
$$

where $W_{r}(\tau)$ is the amplitude pattern in range direction, $\tau$ is the range time (the fast time), $t_{m}$ is the slow time and $\varphi(\tau)$ is the phase of transmitter.

The received signal with time synchronization errors can be written as:

$$
s_{R}\left(\tau, t_{m}\right)=W_{r}\left(\tau-\tau_{d}-e\left(t_{m}\right)\right) \exp \left(j \varphi\left(\tau-\tau_{d}-e\left(t_{m}\right)\right)\right),
$$

where $\tau_{d}=R(t) / c . e\left(t_{m}\right)$ is the time synchronization error, which is defined by:

$$
e\left(t_{m}\right)=\Delta t_{m}+k_{t m} t_{m}+\operatorname{rand}\left(t_{m}\right),
$$

where $\Delta t_{m}$ is the constant time synchronization error. $k_{t m} t_{m}$ is the linear time synchronization error because the PRIs of the transmitter and that of the receiver are not completely equal. As the echoes are received one by one, the linear error will increase gradually. $\operatorname{rand}\left(t_{m}\right)$ is the random time synchronization error which is independent from pulse to pulse. Therefore, it will not accumulate when the azimuth signals are accumulated coherently.

2) Frequency synchronization errors

Suppose phase argument of the transmitted signal is:

$$
\phi_{T}\left(t_{m}, \tau\right)=2 \pi\left(f_{0}+\Delta f_{T}\right) t_{m}+\varphi_{T}\left(t_{m}\right) .
$$

The first term in (4) is the carrier frequency and the second term is the phase noise [12]. The receiver oscillator which has the same form as the transmitter oscillator:

$$
\phi_{R}\left(t_{m}, \tau\right)=2 \pi\left(f_{0}+\Delta f_{R}\right) t_{m}+\varphi_{R}\left(t_{m}\right) .
$$

Thus allowing us to obtain:

$$
\phi\left(t_{m}, \tau\right)=2 \pi \Delta f t_{m}+2 \pi\left(f_{0}+\Delta f_{T}\right) \tau_{d}+\varphi\left(t_{m}\right),
$$

where $\Delta f=\Delta f_{T}-\Delta f_{R}, \varphi\left(t_{m}\right)=\varphi_{T}\left(t_{m}-\tau_{d}\right)-\varphi_{R}\left(t_{m}\right)$.

Because $\Delta f_{T} \ll f_{0}$, so that (6) can be written as:

$$
\phi\left(t_{m}, \tau\right)=2 \pi \Delta f t_{m}+2 \pi f_{0} \tau_{d}+\varphi\left(t_{m}\right) .
$$

The first term is a constant frequency synchronization error, which will result in focused image with a drift. The second term forms the usual Doppler term as bistatic range to the target varies. The last term represents the phase noise in BiSAR system.

As can be seen from (7), the constant frequency offset introduces a phase error related to slow time; meanwhile, the phase noise is independent from pulse to pulse and irrelevant to the positions of targets.

\subsection{Echo Model in Time Domain}

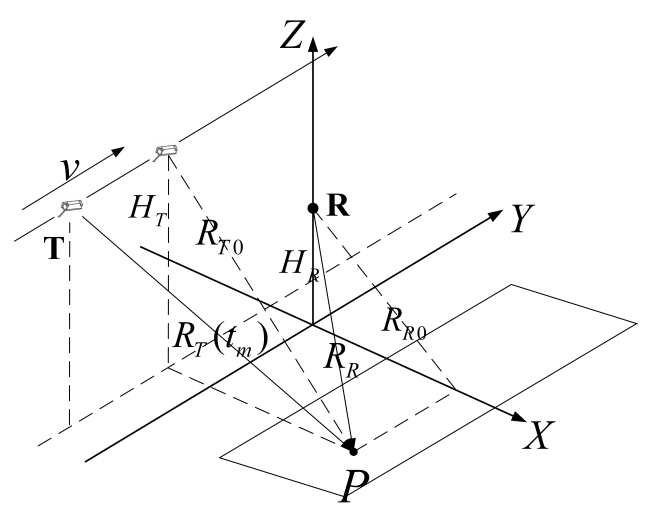

Fig. 1. Geometry of fixed-receiver BiSAR.

Figure 1 shows the imaging geometry of fixed-receiver BiSAR. The origin of the Cartesian coordinates is setting at the projection of the fixed-receiver. The transmitter moves 
with velocity $v$ along the $\mathrm{Y}$ axis, working in stripmap mode. The height of the fixed-receiver is $H_{R}$.

As shown in Fig. 1, the transmitter is at $\left(x_{T}, v t_{m}, H_{T}\right)$ and the fixed-receiver is at $\left(0,0, H_{R}\right)$. The coordinate of point target $P$ is $\left(x_{p}, y_{p}, 0\right)$. Then the bistatic range of point target $P$ can be written as:

$$
R\left(t_{m}, x_{p}, y_{p}\right)=R_{T}\left(t_{m}, x_{p}, y_{p}\right)+R_{R}\left(x_{p}, y_{p}\right),
$$

where

$$
\begin{gathered}
R_{R}\left(x_{p}, y_{p}\right)=\sqrt{H_{R}^{2}+x_{p}^{2}+y_{p}^{2}}, \\
R_{T}\left(t_{m}, x_{p}, y_{p}\right)=\sqrt{R_{T 0}^{2}+v^{2}\left(t_{m}-t_{0}\right)^{2}}, \\
R_{T 0}=\sqrt{H_{T}^{2}+\left(x_{p}-x_{T}\right)^{2}}, t_{0}=y_{p} / v .
\end{gathered}
$$

As can be seen from (9), $R_{R}\left(x_{p}, y_{p}\right)$ is irrelevant to slow time, so the Doppler frequency is only provided by the transmitter in fixed-receiver BiSAR.

According to (2) and (7), the baseband of the scene raw data with time and frequency synchronization errors can be expressed as:

$$
\begin{aligned}
s\left(\tau, t_{m}\right)= & \sum \sigma\left(x_{p}, y_{p}\right) W_{r}\left(\tau-\tau_{p}-e\left(t_{m}\right)\right) W_{a}\left(t_{m}\right) \\
& \times \exp \left(j \pi k_{r}\left(\tau-\tau_{p}-e\left(t_{m}\right)\right)^{2}\right) \exp \left(j \varphi\left(t_{m}\right)\right) \\
& \times \exp \left(-j 2 \pi f_{0} \tau_{p}\right) \exp \left(j 2 \pi \Delta f t_{m}\right),
\end{aligned}
$$

where $\sigma\left(x_{p}, y_{p}\right)$ is the terrain reflectivity at $P, f_{0}$ is the carrier frequency, $k_{r}$ is the chirp rate, $W_{a}\left(t_{m}, x_{p}, y_{p}\right)$ and $W_{r}\left(\tau, x_{p}, y_{p}\right)$ describe the antenna beam patterns in azimuth and range dimension, respectively.

According to (12), the linear time synchronization error introduces an increasing displacement of echoes in range direction along slow time. The constant frequency synchronization error introduces a phase error related to slow time. As the echoes are received one by one, the displacement and phase error will increase gradually with slow time. Then these two kinds of synchronization errors can be added during 2-D inverse Stolt transform in 2-D frequency domain. The constant and random time synchronization errors and phase noise are independent from pulse to pulse and irrelevant to the positions of targets. So these three kinds of synchronization errors can be added by phase multiplication in time domain.

Therefore, raw data simulation for fixed-receiver BiSAR with time and frequency synchronization errors employs a procedure that consists of two main stages. In the first stage, raw data with linear time and constant frequency synchronization errors is generated in 2-D frequency domain. In the second stage, constant and random time synchronization errors and phase noise are introduced by phase multiplication in time domain. Scene raw data can be seen as convolution between the matrix of terrain reflectivity and transfer function of the SAR system. With regard to the first stage, the
SAR system 2-D pulse response must be properly changed in the existence of linear time and constant frequency synchronization errors.

\subsection{Echo Model in 2-D Frequency Domain}

Scene raw data with linear time and constant frequency synchronization errors can be written as:

$$
\begin{aligned}
s\left(\tau, t_{m}\right)= & \sum \sigma\left(x_{p}, y_{p}\right) W_{r}\left(\tau-\tau_{p}-k_{t m} t_{m}\right) W_{a}\left(t_{m}\right) \\
& \times \exp \left(j \pi k_{r}\left(\tau-\tau_{p}-k_{t m} t_{m}\right)^{2}\right) \\
& \times \exp \left(-j 2 \pi f_{0} \tau_{p}\right) \exp \left(j 2 \pi \Delta f t_{m}\right) .
\end{aligned}
$$

After 2-D Fourier transform, thus allowing us to obtain the 2-D frequency spectrum of (13):

$$
\begin{aligned}
S\left(f_{a}, f_{\tau}\right)= & \sum \sigma\left(x_{p}, y_{p}\right) W_{r}\left(f_{\tau}\right) W_{a}\left(f_{a}\right) \exp \left(-j \pi \frac{f_{\tau}^{2}}{k_{r}}\right) \\
& \times \exp \left(-j 2 \pi\left(f_{a} t_{0}+f_{0} \tau_{p}\right)\right) \\
& \times \exp \left(-j 2 \pi\left(R_{R} \frac{f_{\tau}+f_{0}}{c}+R_{T 0} \cdot F_{1}\right)\right),
\end{aligned}
$$

where $f_{\tau}$ is the frequency variable corresponding to $\tau, f_{a}$ is the frequency variable corresponding to $t_{m} . F_{1}$ is a $2-\mathrm{D}$ coupled term, which is described by:

$$
F_{1}=\sqrt{\left(\frac{f_{\tau}+f_{0}}{c}\right)^{2}-\left(\frac{f_{a}+k_{t m}+\Delta f}{v}\right)^{2}} .
$$

The phase of (14) can be divided into three exponential parts: the first part is the frequency spectrum of transmitted chirp signal; the second exponential term is the phase related to the azimuth position of $P$, and the last exponential term is the phase related to 2-D spatial variation in fixed-receiver BiSAR.

For monostatic SAR and translational invariant BiSAR, phases of $R_{R}$ and $R_{T 0}$ have the same square root expression in 2-D frequency domain, which can be acquired simultaneously through 2-D inverse Stolt transform in 2-D frequency domain. However, as can be seen from the last part in (14), the difference in velocity between the transmitter and the receiver produces 2 -D spatial variation in range and azimuth dimensions. So the phase of $R_{R}$ and $R_{T 0}$ cannot be obtained simultaneously in 2-D frequency domain. How to get (14) in 2-D frequency domain is the key problem to realize 2-D spatial variation simulation for fixed-receiver BiSAR with linear time and constant frequency synchronization errors.

\section{Rapid Raw Data Simulation}

Raw data simulation for fixed-receiver BiSAR can be divided into two parts: one is simulation of the terrain reflectivity; the other is to establish the model of transfer function of SAR system. A great deal of work has been done by plenty of researches in the the simulation of terrain reflectivity $[13,14]$. In this paper, the main work is to establish the 
model of transfer function of SAR system. Therefore, we use the general method to compute the scattering coefficient in this paper, which means that we start with a reflectivity map as an input.

Firstly, the terrain reflectivity of the simulated scene is projected to the slant range plane. Then, each sampling point is multiplied by the phase related to bistatic range, so the expression can be written as:

$$
s^{\prime}\left(\tau, t_{m}\right)=\sum \sigma\left(x_{p}, y_{p}\right) \exp \left(-j 2 \pi f_{0} \frac{R_{T 0}+R_{R}}{c}\right)
$$

after 2-D Fourier transform, thus allowing us to obtain:

$$
\begin{aligned}
S\left(f_{a}^{\prime}, f_{\tau}^{\prime}\right)= & \sum \sigma\left(x_{p}, y_{p}\right) \exp \left(-j 2 \pi f_{a}^{\prime} t_{0}\right) \\
& \times \exp \left(-j 2 \pi \frac{f_{\tau}^{\prime}+f_{0}}{c}\left(R_{R}+R_{T 0}\right)\right),
\end{aligned}
$$

where $f_{a}^{\prime}$ and $f_{\tau}^{\prime}$ is the azimuth and range frequency variables in 2-D frequency domain, respectively.

The conversion of inverse Stolt transform is as follows:

$$
\left\{\begin{array}{l}
f_{a}^{\prime}=f_{a}, \\
f_{\tau}^{\prime}=c \cdot F_{1}-f_{0},
\end{array}\right.
$$

thus obtaining:

$$
\begin{aligned}
S\left(f_{a}, f_{\tau}\right)= & \sum \sigma\left(x_{p}, y_{p}\right) \exp \left(-j 2 \pi f_{a} t_{0}\right) \\
& \times \exp \left(-j 2 \pi\left(R_{R}+R_{T 0}\right) \cdot F_{1}\right) .
\end{aligned}
$$

Comparing (19) and (14), it can be found that the phase related to $R_{R}$ in (19) changes with $f_{a}$, but the anticipative phase related to $R_{R}$ in (14) has no relations with $f_{a}$. So (19) cannot achieve 2-D spatial variation simulation for fixedreceiver BiSAR. We define $\Theta\left(R_{R}, f_{a}, f_{\tau}\right)$ as:

$$
\Theta\left(R_{R}, f_{a}, f_{\tau}\right)=\exp \left(-j 2 \pi R_{R}\left(\frac{f_{\tau}+f_{0}}{c}-F_{1}\right)\right) .
$$

Substituting (20) into (19) allows us to obtain:

$$
\begin{aligned}
S\left(f_{a}, f_{\tau}\right)= & \sum \sigma\left(x_{p}, y_{p}\right) \exp \left(-j \pi \frac{f_{\tau}^{2}}{k_{r}}\right) \exp \left(-j 2 \pi f_{a} t_{0}\right) \\
& \times \exp \left(-j 2 \pi\left(R_{R} \frac{f_{\tau}+f_{0}}{c}+R_{T 0} F_{1}\right)\right) \\
& \times \exp \left(j \Theta\left(R_{R}, f_{a}, f_{\tau}\right)\right)
\end{aligned}
$$

According to (21) and (14), it can be found that there is an additional differential phase $\Theta\left(R_{R}, f_{a}, f_{\tau}\right)$. As $\Theta\left(R_{R}, f_{a}, f_{\tau}\right)$ is related to $R_{R}$ and changes with $f_{a}$ and $f_{\tau}$. If the phase of reference point target (middle point target) $\Phi_{\text {ref }}$ is taken to compensate the additional phase in 2-D frequency domain, the residual phase errors of marginal point targets are shown in Fig. 2 (simulation parameters are listed in Tab. 2).

After phase compensation in 2-D frequency domain, Fig. 2 illustrates the residual phase errors of marginal point targets (near and far targets). We can see clearly that the residual phase errors of marginal targets are more than $12 \mathrm{rad}$, then the marginal targets will be totally unfocused in azimuth focusing. So the additional differential phase $\Theta\left(R_{R}, f_{a}, f_{\tau}\right)$ cannot be compensated in 2-D frequency domain. To resolve the range-azimuth frequency coupling of the receiver in (20), inverse Fourier transform of the range frequency $f_{\tau}$ is performed. Then the range-Doppler frequency spectrum of (17) can be written as follows:

$$
\begin{aligned}
S\left(\tau, f_{a}\right)= & \sum \sigma\left(x_{p}, y_{p}\right) \exp \left(-j 2 \pi f_{a} t_{0}\right) \\
& \times \exp \left(-j 2 \pi\left(\frac{R_{T 0}+R_{R}}{c} \cdot F_{2}\right)\right),
\end{aligned}
$$

where $F_{2}$ is described by:

$$
F_{2}=\sqrt{f_{0}^{2}-\left(\frac{c\left(f_{a}+k_{t m}+\Delta f\right)}{v}\right)^{2}} .
$$

Meanwhile, by omitting the linear frequency modulation, the expression of (14) in the range-Doppler frequency domain can be written by:

$$
\begin{aligned}
S\left(\tau, f_{a}\right)= & \sum \sigma\left(x_{p}, y_{p}\right) \exp \left(-j 2 \pi f_{a} t_{0}\right) \\
& \times \exp \left(-j 2 \pi\left(\frac{R_{R}}{\lambda}+\frac{R_{T 0}}{c} F_{2}\right) .\right.
\end{aligned}
$$

According to (22) and (24), the additional phase in range-Doppler frequency domain is:

$$
\Phi_{\Delta}=\exp \left(-j 2 \pi R_{R}\left(\frac{1}{\lambda}-\frac{F_{2}}{c}\right)\right) .
$$

According to (25), the additional phase $\Phi_{\Delta}$ changes slowly with $f_{a}$. When $\Phi_{\Delta}$ is compensated by the phases of reference targets (azimuth: 0) in range-Doppler frequency domain, the residual phase errors of the marginal targets (azimuth: +1000 m, +5000 m) are shown in Fig. 3.

As can be seen from Fig. 3(b), after phase compensation in range-Doppler frequency domain, the residual phase errors of the marginal targets are quadratic modulation phases. When the size of the simulated scene is large, the quadratic modulation phase errors may be significant, which is shown in Fig. 3(b).

According to the azimuth compression theory [3], the quadratic modulation phase error can cause dissymmetry of sidelobes, high PSLR and ISLR, etc. When $\pi / 4 \mathrm{rad}$ is used as the the residual phase error lower bound in azimuth dimension (quadratic phase error: $|\mathrm{QPE}| \leq \pi / 4 \mathrm{rad}$ ), the azimuth width of the simulated scene should satisfy:

$$
\left|2 \pi\left(R_{R}-R_{R r e f}\right)\left(\frac{1}{\lambda}-\frac{F_{2}}{c}\right)\right|<\frac{\pi}{4} .
$$

For the simulation parameters listed in Tab. 2, the azimuth width is confined by:

$$
X \leq 7853 \mathrm{~m} .
$$

In this paper, through phase compensation of (25) in range-Doppler domain, the 2-D range-azimuth frequency coupling of the receiver can be resolved. After multiplied by the linear frequency modulation $\exp \left(-j \pi f_{\tau}^{2} / k_{r}\right)$, antenna beam pattern in range and azimuth directions, constant 


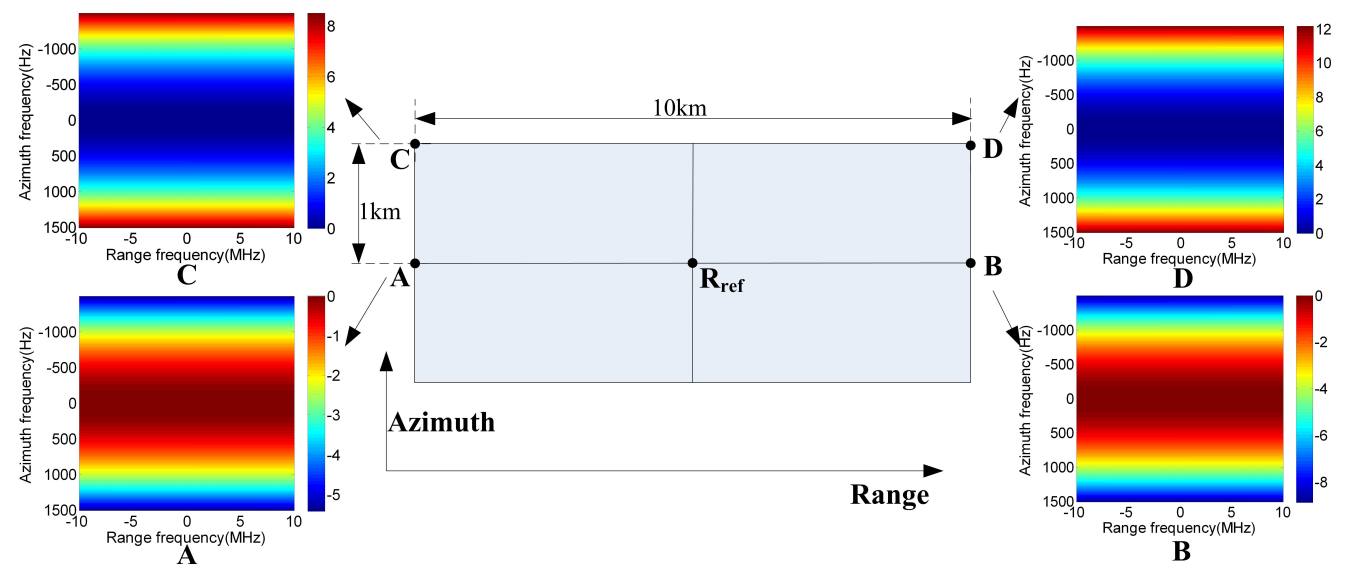

Fig. 2. Residual phase errors after phase compensation in 2-D frequency domain.

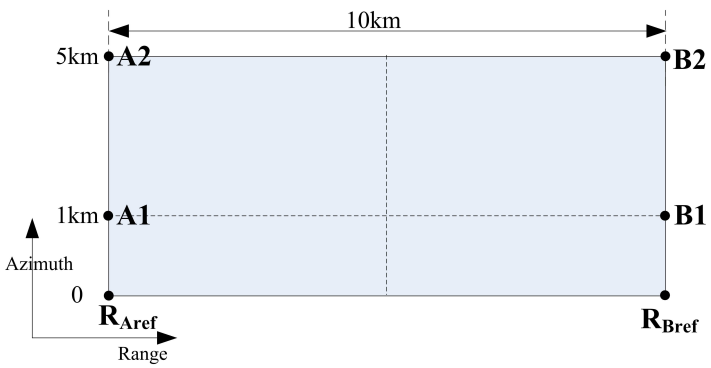

(a)

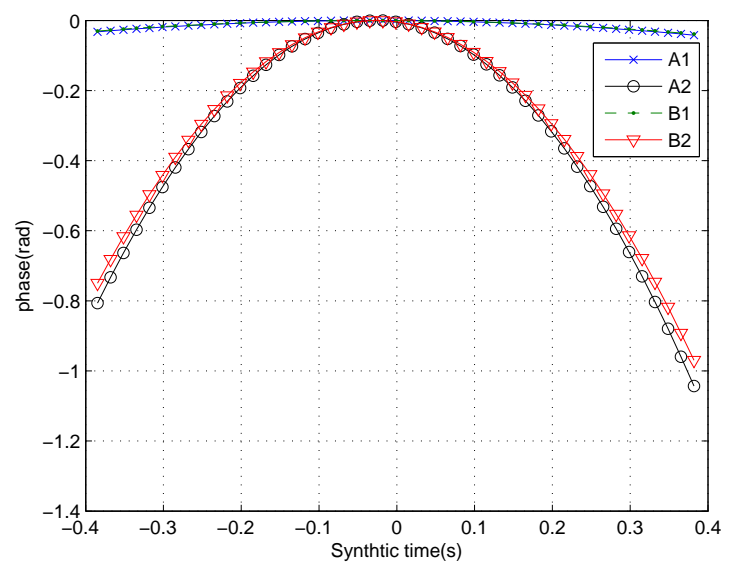

(b)

Fig. 3. Residual phase errors of marginal targets. (a) Marginal targets; (b) Residual phase errors.

and random time synchronization errors $\exp \left(-j 2 \pi f_{\tau}\left(\Delta t_{m}+\right.\right.$ $\left.\left.\operatorname{rand}\left(t_{m}\right)\right)\right)$ and phase noise $\exp \left(j\left(\varphi\left(t_{m}\right)\right)\right)$, we can get the anticipative 2-D frequency spectrum of scene raw data with time and frequency synchronization errors.

Therefore, the proposed method of rapid raw data simulation for fixed-receiver BiSAR is described as follows:

1) According to the geometry of fixed-receiver BiSAR system, surface reflectivity of the scene is projected to slantrange plane.
2) A phase term related to bistatic range is multiplied to the surface reflectivity, which is shown in (16).

3) After 2-D Fourier transform, 2-D frequency spectrum of (16) is presented in (17).

4) The phase of reference point target (28) is multiplied to (17)

$$
H_{1}\left(f_{\tau}, f_{a}\right)=\exp \left(j 2 \pi \frac{f_{\tau}+f_{0}}{c}\left(R_{R r e f}+R_{\text {Tref }}\right)\right) .
$$

After multiplied by the reference phase, high frequency modulation phase in (17) is changed into a baseband modulation phase. So the inverse Stolt transform is realized in baseband, and the high frequency modulation in the center frequency can be neglected [9].

5) Based on (17), 2-D inverse Stolt transform is performed.

6) After range inverse Fourier transform, the additional phase is compensated by the phase of reference point target in rangeDoppler frequency domain.

7) After multiplied by the phase of transmit chirp $H_{2}\left(f_{\tau}, f_{a}\right)$ and the reference phase $H_{3}\left(f_{\tau}, f_{a}\right)$ in 2-D frequency domain, we can get the anticipative 2-D frequency spectrum of scene raw data with linear time and constant frequency synchronization errors.

$$
\begin{gathered}
H_{2}\left(f_{\tau}, f_{a}\right)=W_{r}\left(f_{\tau}\right) W_{a}\left(f_{a}\right) \exp \left(-j \pi \frac{f_{\tau}^{2}}{k_{r}}\right), \\
H_{3}\left(f_{\tau}, f_{a}\right)=\exp \left(-j 2 \pi\left(R_{R r e f} \frac{f_{\tau}+f_{0}}{c}+R_{\text {Tref }} F_{1}\right)\right) .
\end{gathered}
$$

8) After inverse Fourier transform in azimuth direction, phase of constant and random time synchronization errors $H_{T}\left(f_{\tau}, t_{m}\right)$ is multiplied to (14).

$$
H_{T}\left(f_{\tau}, t_{m}\right)=\exp \left(-j 2 \pi f_{\tau}\left(\Delta t_{m}+\operatorname{rand}\left(t_{m}\right)\right)\right) .
$$

9) Finally, after inverse Fourier transform in range direction and multiplied by the phase noise of (32), scene raw data of fixed-receiver BiSAR with time and frequency synchronization errors can be obtained.

$$
H_{F}\left(\tau, t_{m}\right)=\exp \left(j\left(\varphi\left(t_{m}\right)\right)\right)
$$




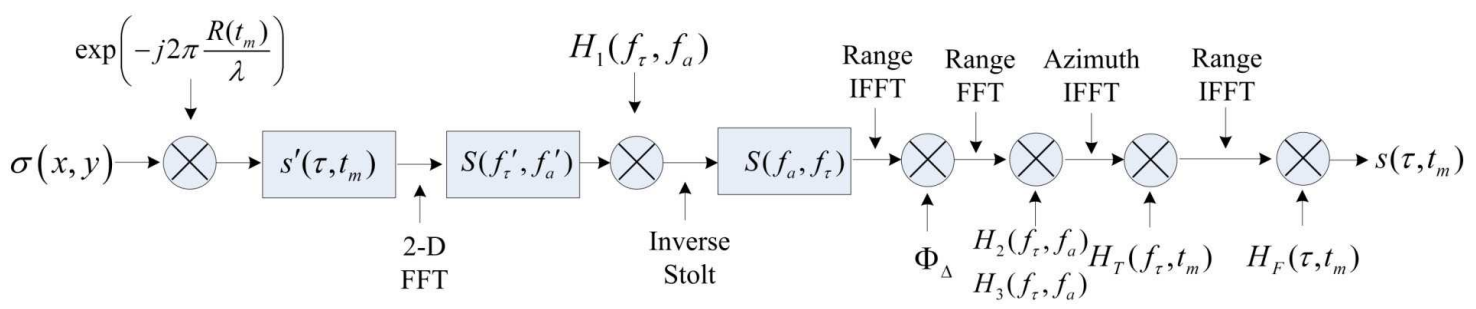

Fig. 4. Scheme of the proposed method.

The proposed method can be performed along the scheme shown in Fig. 4.

The computational complexity of the TBT method and the proposed method are evaluated in this section. The main operations of the raw data simulation method are complex multiplications and additions. Due to the fact that the complex multiplications consume much more resources and time of the computer than the complex additions, the complex multiplications is used to indicate the computational complexity of the TBT method and the proposed method. We assume that $N_{r}$ and $N_{a}$ are the sample units in range and azimuth dimensions (in pixels) of the final raw data. The target numbers in range and azimuth dimensions are denoted by $M_{r}$ and $M_{a}$, respectively. The number $N_{S C}$ of complex multiplications needed by terrain reflectivity computation can be written as $[13,14]$ :

$$
N_{S C}=0.5 M_{a} M_{r}^{2}+90.5 M_{a} M_{r}-85 M_{a}-86 M_{r}+172 .
$$

As the raw data of the simulated scene is calculated target by target and pulse by pulse in 2-D time domain, so the complex multiplications of each target is $N_{r} N_{a}$. The number $N_{T D}$ of complex multiplications needed by the TBT method can be expressed as:

$$
N_{T D}=N_{a} M_{a} M_{r} N_{r} \cdot C M+N_{S C},
$$

where $C M$ denote the complex multiplications.

By analyzing Fig. 4 , the number $N_{F D}$ of complex multiplications needed by the proposed method is described by:

$$
\begin{aligned}
N_{F D}= & 2\left(N_{a} N_{r} \log _{2} N_{r}+N_{r} N_{a} \log _{2} N_{a}\right) \cdot B C \\
& +N_{a} N_{r}(k+6) \cdot C M+N_{S C},
\end{aligned}
$$

where $B C$ denotes the butterfly calculation in FFT, and $k$ is the order of Stolt interpolation.

A butterfly calculation equals to ten floating-point operations, and a complex multiplications equals to six floatingpoint operations [15]. Then (34) and (35) can be rewritten as:

$$
N_{T D}=6 N_{a} M_{a} M_{r} N_{r}+N_{S C}
$$

$$
N_{F D}=N_{a} N_{r}\left(20 \log _{2} N_{r}+20 \log _{2} N_{a}+6 k+36\right)+N_{S C} .
$$

According to (36) and (37), the computational complexity of the two methods are computed and shown in Fig. 5.

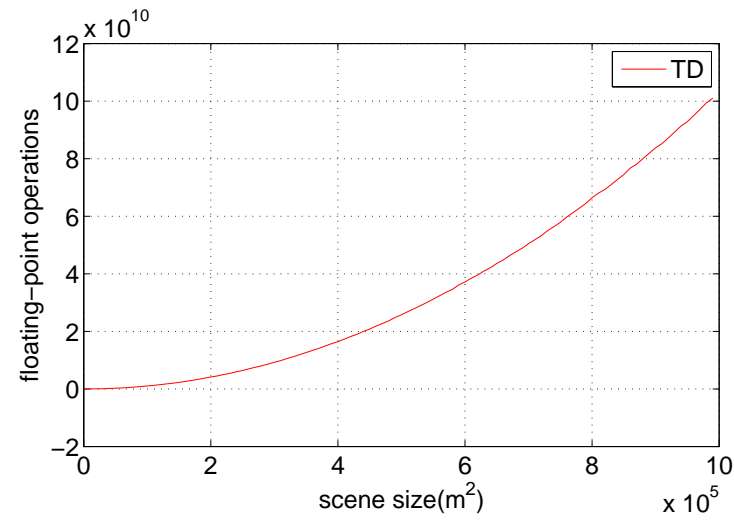

(a)

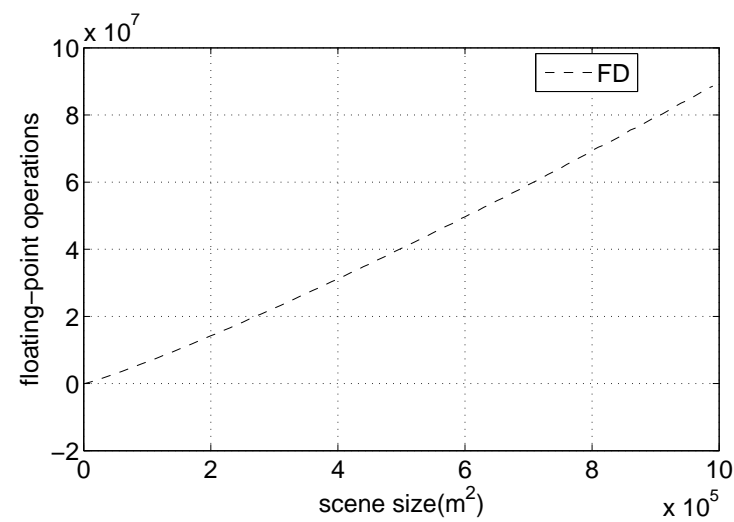

(b)

Fig. 5. Computational complexity. (a) TBT method; (b) proposed method.

Figure 5 shows the number of floating-point operation needed by the proposed method and the TBT method, respectively. The floating-point operation of the TBT method is shown in Fig. 5(a). The order of the magnitude is $10^{10}$. The floating-point operation of the proposed algorithm is shown in Fig. 5(b). The order of the magnitude is $10^{7}$. By comparing the floating-point operations of the two methods, it can be found that the proposed algorithm is remarkably efficient with floating-point operation measured to be at least of 3 orders of magnitude less than the TBT method, especially when large size of scene is simulated.

\section{Simulation Results}

Several simulations are performed in this section to verify the proposed rapid raw data simulation approach. First, 


\begin{tabular}{|c|c|c|c|}
\hline Parameter & Value & Parameter & Value \\
\hline$\Delta t_{m}$ & $0.02 \mathrm{~ns}$ & $\Delta f$ & $100 \mathrm{~Hz}$ \\
\hline$k_{t m}$ & $1 \times 10^{-9}$ & $\sigma_{y}^{2}(1 \mathrm{~s})$ & $\times 10^{-11}$ \\
\hline $\operatorname{rand}\left(t_{m}\right)$ & $1 \times 10^{-8}$ & & \\
\hline
\end{tabular}

Tab. 1. Time and frequency synchronization error.

\begin{tabular}{|c|c|c|}
\hline Parameter & Tx & Rx \\
\hline Height $(\mathrm{km})$ & 514 & 10 \\
\hline Incline angle $\left(^{\circ}\right)$ & 45 & 60 \\
\hline Antenna dimension $\left(^{\circ}\right)$ & $1.5 \times 0.33$ & $15 \times 2.3$ \\
\hline Velocity $(\mathrm{m} / \mathrm{s})$ & 7600 & 0 \\
\hline Center freq $(\mathrm{GHz})$ & 9.65 & 9.65 \\
\hline PRF $(\mathrm{Hz})$ & 3000 & \\
\hline Chirp duration $(\mu \mathrm{s})$ & 10 & \\
\hline Bandwidth $(\mathrm{MHz})$ & 50 & \\
\hline Sample freq $(\mathrm{MHz})$ & & 100 \\
\hline
\end{tabular}

Tab. 2. Simulation parameters

raw data of point targets is generated both by the proposed method and the TBT method. Simulation results are compared to validate the proposed algorithm. Second, extended scene simulation results of fixed-receiver BiSAR are presented to verify the efficiency of the proposed method.

\subsection{Point Target Simulation}

The parameters of time and frequency synchronization errors [10] are listed in Tab. 1, and the simulation parameters are listed in Tab. 2. Firstly, we consider a scene consisting of a single point scatter placed in the center of the scene. For such a scene, it is possible to compare the raw signal simulated via the proposed method to the TBT one.

Figure 6 shows the amplitudes of the simulated echo generated by the TBT method and the proposed method. Due to the linear time synchronization error, there is an obvious displacement of echoes in range direction and the displacement increases gradually with slow time. To compare the detail of the echoes generated by the two methods, the unitary amplitudes of the echoes at some range and azimuth lines are illustrated in Fig. 7(a) and (b), respectively. In the range dimension, the chirp duration of the proposed method match the chirp duration of the TBT method quite well, in general. The Fresnel undulation of the echoes in the range dimension is caused by multiplying transmit chirp signal in the range frequency domain [15]. In the azimuth dimension, the synthetic aperture time of the proposed method and the TBT method are almost the same. Because of Sinc interpolation in azimuth dimension, amplitude of the echo generated by the proposed method is deviate from rectangle.

Secondly, to verify the wide scene simulation ability of the proposed method, raw data simulation of several point targets in different positions for fixed-receiver BiSAR is performed. The size of simulated scene is $2 \mathrm{~km} \times 4 \mathrm{~km}$. After synchronization processing $[1,16]$ and focused by bistatic imaging algorithm [3,17], the image is shown in Fig. 8(a). It can be clearly seen from Fig. 8(a) that the point targets are all well focused.

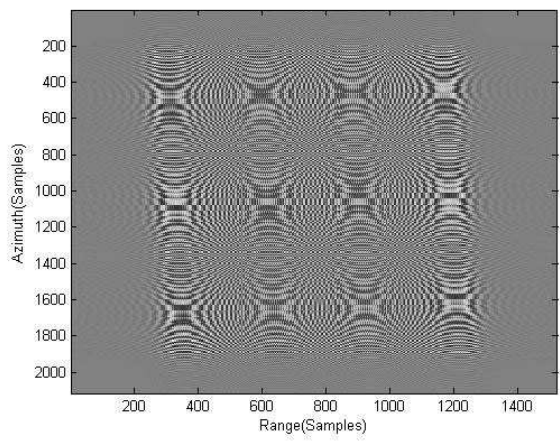

(a)

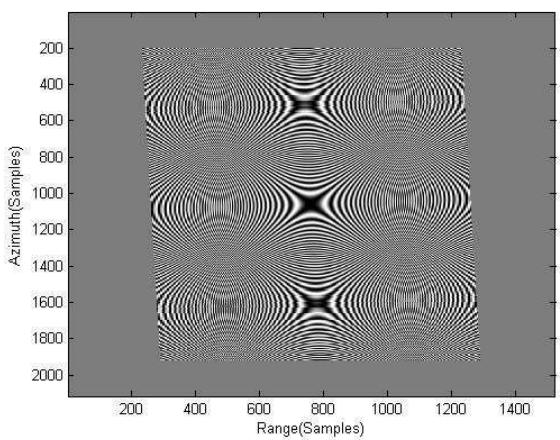

(b)

Fig. 6. Simulated SAR raw data. (a) Proposed method; (b) TBT method.

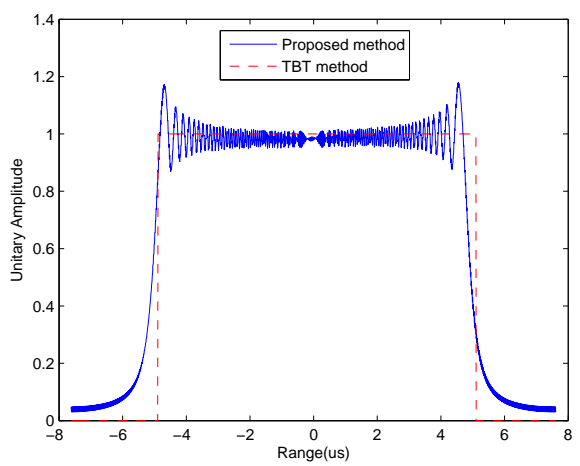

(a)

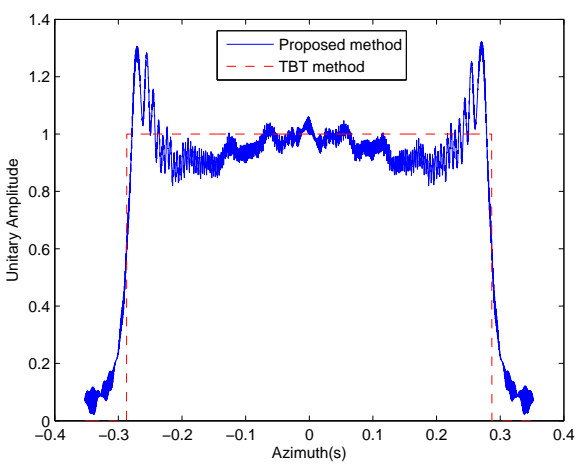

(b)

Fig. 7. Amplitude of the simulated signal in the time domain. (a) Range direction; (b) azimuth direction. 


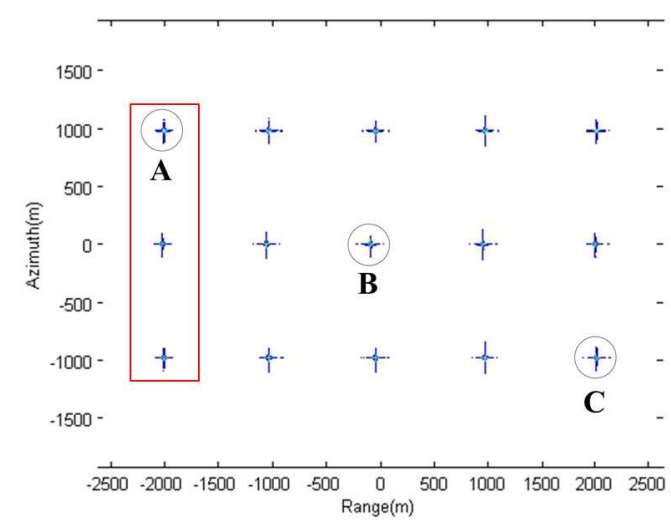

(a)

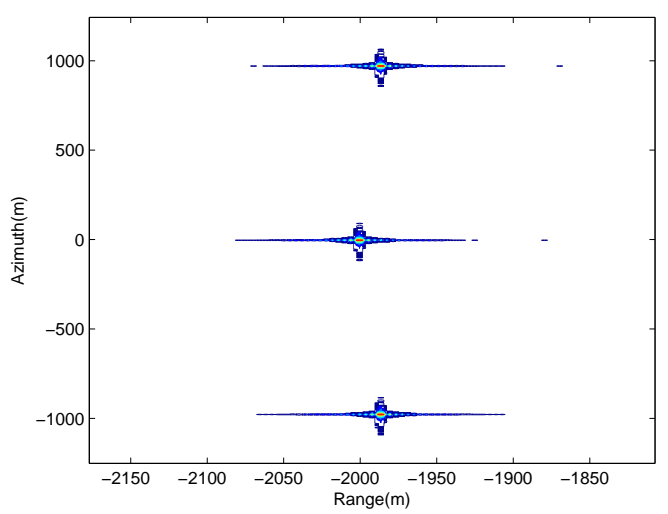

(b)

Fig. 8. Images of point target. (a) Image of extended scene; (b) 2-D spatial variation.

From Fig. 8(b) we can find that the three targets located at same range bins but different azimuth positions will have different range histories (range delay). So the 2-D spatial variation in fixed-receiver BiSAR occurs as predicted. Meanwhile, image performances of three representative point targets (A, B and C) are listed in Tab. 3.

\begin{tabular}{|c|c|c|c|c|}
\hline Parameter & Ideal & A & B & C \\
\hline Azimuth resolution (m) & 2.66 & 2.78 & 2.71 & 2.69 \\
\hline Azimuth PSLR (dB) & -13.26 & -13.31 & -13.27 & -13.9 \\
\hline Range resolution (m) & 3.54 & 3.61 & 3.58 & 3.63 \\
\hline Range PSLR (dB) & -13.26 & -12.9 & -13.1 & -12.5 \\
\hline
\end{tabular}

Tab. 3. Image performances.

As can be seen from Tab. 3, the range and azimuth resolutions of the three representative point targets are approaching the ideal values quite well. The PSLR in the azimuth dimension is a little high, which is caused by the residual quadratic modulation phase error. But it has no evident influence on image focusing, so we do not make further research on it in this paper.

\subsection{Scene Echo Simulation}

A realistic scene raw data simulation for fixed-receiver BiSAR is performed to verify the feasibility and efficiency of the proposed raw data simulation method. The realistic scene is about $5 \mathrm{~km}$ (azimuth) $\times 5 \mathrm{~km}$ (range). The DEM of the realistic scene is depicted in Fig. 9(a). The scattering coefficient is shown in Fig. 9(b).

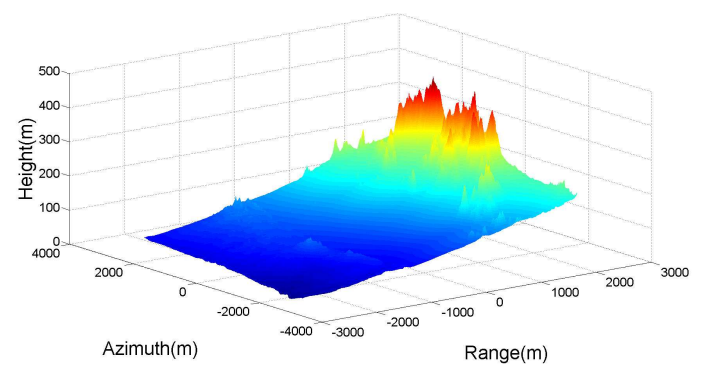

(a)

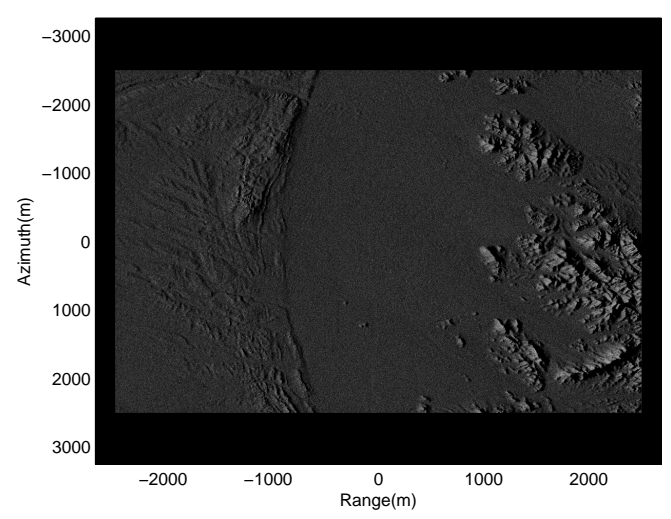

(b)

Fig. 9. Extended scene simulation. (a) DEM of the simulated scene; (b) scattering coefficient.

Scene raw data are focused by bistatic imaging algorithm $[3,17]$. Images with and without synchronization procedure of $[1,16]$, are reported in Fig. 10(a) and (b), respectively.

From Fig. 10(a), we can conclude that time and frequency synchronization errors will result unfocused images, drift of radar echoes and displacement of targets. To focus BiSAR raw data, time and frequency synchronization compensation procedure must be applied. From Fig. 10(b), it is clear that the image fit very well with the realistic scene after time and frequency synchronization. Meanwhile, speckle noise and foreseen geometric distortion (foreshortening) are apparent. Furthermore, time consumed by the proposed method in a personal computer (PC configuration: Intel CORE i5, $3.2 \mathrm{GHz}, 4 \mathrm{~GB}$ memory) is only some dozens minutes, which is much less than the TBT method (several thousand minutes). On the whole, the proposed method has greater efficiency for scene raw data simulation with time and frequency synchronization errors.

\section{Conclusion}

In this paper, an efficient rapid raw data simulation approach for fixed-receiver BiSAR with time and frequency 


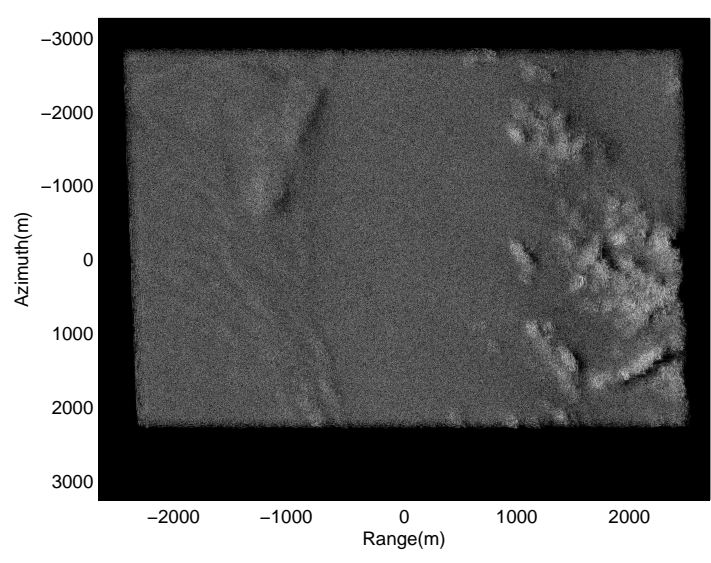

(a)

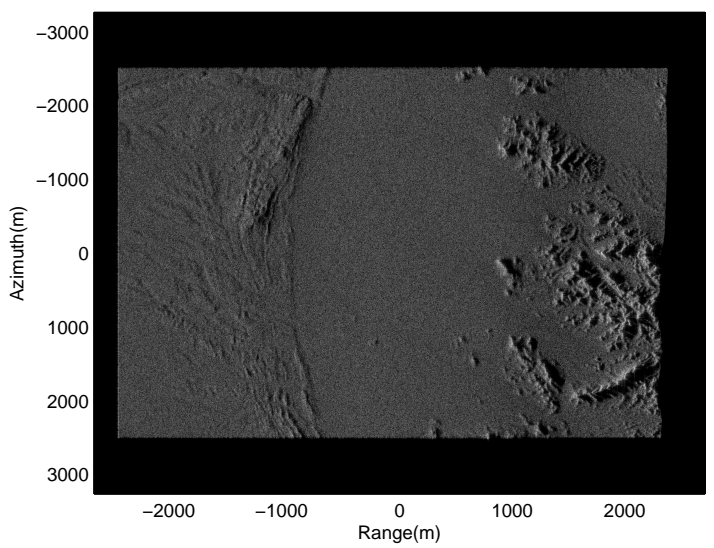

(b)

Fig. 10. Images of simulated scene. (a) Without synchronization; (b) with synchronization.

synchronization errors is presented. Based on the 2-D inverse Stolt transform in 2-D frequency domain and phase compensation in range-Doppler frequency domain, the efficiency of scene raw data simulation for fixed-receiver BiSAR is greatly improved. Point targets and scene raw data are simulated both by the TBT method and the proposed method, respectively. Simulation results prove that the proposed rapid raw data simulation method is much more efficient than the TBT method. However, as the fixed-receiver BiSAR is a special kind of translational variant BiSAR system, the proposed method cannot be directly used in other translational variant BiSAR system. The rapid raw data simulation methods for translational variant BiSAR need further study in the future.

\section{Acknowledgments}

This work was partly supported by National Natural Science Foundation of China (Grant Nos. 61501477), the research project of National University of Defense Technology (Grant No. CJ12-04-02).

\section{References}

[1] WANG, W. Q., DING, C. B., LIANG, X. D. Time and phase synchronisation via direct-path signal for bistatic synthetic aperture radar systems. IET Radar, Sonar \& Navigation, 2008, vol. 2, no. 1, p. 1-11. ISSN: 1751-8784. DOI: 10.1049/iet-rsn:2006 0097

[2] QIU, X. L., HU, D. H., ZHOU, L., et al. A bistatic SAR raw data simulator based on inverse omega-k algorithm. IEEE Transactions on Geoscience and Remote Sensing, 2010, vol. 48, no. 3, p. 1540-1547. ISSN: 0196-2892. DOI: 10.1109/TGRS.2009.2032776

[3] ZHANG, Q. L., CHANG, W. G., LI, X. Y. An extended NLCS algorithm for bistatic fixed-receiver SAR imaging. In Proceedings of the 7th European Radar Conference. Paris (France), 2010, p. 252-255. DOI: $10.1109 / \mathrm{EuR} .2010 .843$

[4] ZHANG, F., HU, C., LI, W., et al. Accelerating time-domain SAR raw data simulation for large areas using multi-GPUs. IEEE Journal of Selected Topics in Applied Earth Observations and Remote Sensing, 2014, vol. 7, no. 9, p. 3956-3966. ISSN: 1939-1404. DOI: 10.1109/JSTARS.2014.2330333

[5] FRANCESCHETTI, G., IODICE, A., PERNA, S., et al. Efficient simulation of airborne SAR raw data of extended scenes. IEEE Transactions on Geoscience and Remote Sensing, 2006, vol. 44, no. 10, p. 2851-2860. ISSN: 0196-2892. DOI: 10.1109/TGRS.2006.875786

[6] PERNA, S., FRANCESCHETTI, G., IODICE, A., et al. SAR sensor trajectory deviations Fourier domain formulation and extended scene simulation of raw signal. IEEE Trans on Geoscience and Remote Sensing, 2006, vol. 44, no. 9, p. 2323-2334. ISSN: 0196-2892. DOI: 10.1109/TGRS.2006.873206

[7] FRANCESCHETTI, G., GUIDA, R., INDICE, A., et al. Simulation tools for interpretation of high resolution SAR images of urban areas. In IEEE Urban Remote Sensing Joint Event. Paris (France), 2007, p. 1-5. DOI: 10.1109/URS.2007.371841

[8] FRANCESCHETTI, G., INDICE, A., NATALE, A., et al. Bistatic SAR simulation: Time and frequency domain approaches. In IEEE International Conference on Digital Signal Processing. Corfu (Greece), 2011, p. 1-7. DOI: 10.1109/ICDSP.2011.6005021

[9] QIU, X. L., HU, D. H., DING, C. B. Bistatic synthetic aperture radar imaging and processing. 1st ed. Beijing (China): Science Press, 2010. ISBN: 9787030270856

[10] TIAN, W. M., LONG, T., YANG, J., et al. Combined analysis of time and frequency synchronization errors for BiSAR. In IEEE CIE International Conference on Radar. Chengdu (China), 2011, p. 388-392. DOI: 10.1109/CIE-Radar.2011.6159559

[11] ZHOU, P., LIN, L. J., DAI, Y. S., et al. Influence of frequency synchronization errors on hybrid bistatic SAR system. In International Conference on Information Science, Electronics and Electrical Engineering. Sapporo (Japan), 2014, p. 1358-1362. DOI: 10.1109/InfoSEEE.2014.6947898

[12] GERHARD, K., MARWAN, Y. Impact of oscillator noise in bistatic and multistatic SAR. IEEE Geoscience and Remote Sensing Letters, 2006, vol. 3, no. 3, p. 424-428. ISSN: 1545-598X. DOI 10.1109/LGRS.2006.874164

[13] REN, S. H., CHANG, W. G., LI, J. Y. Raw signal simulation research of three-dimensional scene with undulate characteristics. Radar Science and Technology, 2007, vol. 5, no. 5, p. 324-328. ISSN: 16722337. DOI: $10.3969 /$ j.issn.1672-2337.2007.05.006

[14] SUN, H. W., ZENG, T., YANG, J. Improvements on SAR simulation of three-dimensional forest canopies. In IET International Radar conference. Xi' an (China), 2013, p. 1-5. DOI: 10.1049/cp.2013.0507

[15] HU, G. S. Digital Signal Processing: Theory. 1st ed. Beijing (China): Tsinghua University Press, 2003. ISBN: 9787302065067 
[16] PACO, L. D., JORDI, J. M., PAU, S. M., et al. Phase synchronization and Doppler centroid estimation in fixed receiver bistatic SAR systems. IEEE Transactions on Geoscience and Remote Sensing, 2008, vol. 46, no. 11, p. 3459-3471. ISSN: 0196-2892. DOI: 10.1109/TGRS.2008.923322

[17] QIU, X. L., HU, D. H., DING, C. B. An improved NLCS algorithm with capability analysis for one-stationary BiSAR. IEEE Transactions on Geoscience and Remote Sensing, 2008, vol. 46, no. 10, p. 31793186. ISSN: 0196-2892. DOI: 10.1109/TGRS.2008.921569

\section{About the Authors ...}

Feifei YAN was born in Henan, P. R. China in 1987. He received the B.S. degree in Electronic Information Engineering from Xidian University, Xian, China, in 2005, and the M.S. degree in Information Engineering from the National University of Defense Technology, Changsha, China, in 2011.
He is currently working towards the Ph. D. degree in Information Engineering at the National University of Defense Technology. His current research interests include bistatic radar systems, and parameter estimation.

Wenge CHANG was born in Shanxi, P. R. China in 1965. He received the M.S. and Ph.D. degrees in Information and Communication Engineering from the National University of Defense Technology, Changsha, China, in 1993 and 2001, respectively. He is currently a Professor with the National University of Defense Technology. His fields of interest include synthetic aperture radar systems and signal processing.

Xiangyang LI was born in Hunan, P. R. China in 1972. He received the M.S. and Ph.D. degrees in Information and Communication Engineering from the National University of Defense Technology, Changsha, China, in 1996 and 2000, respectively. He is currently a Professor with the National University of Defense Technology. His fields of interest includes synthetic aperture radar systems and signal processing. 